\title{
Ellipsometric determination of permittivity in a negative index photonic crystal metamaterial
}

\author{
Principia Dardano ${ }^{1}$, Massimo Gagliardi ${ }^{1}$, Ivo Rendina ${ }^{1}$, Stefano Cabrini ${ }^{2}$ and Vito Mocella ${ }^{1}$ \\ In this work, we present the first experimental evidence of negative dielectric susceptibility in a two-dimensional silicon photonic \\ crystal (PhC) with negative refractive index behavior. In the frequency range in which the effective refractive index $n_{\mathrm{eff}}$ is equal to -1 , \\ the incident light couples efficiently to the guided modes in the top surface layer of the PhC metamaterial. These modes resemble \\ surface plasmon polariton resonances. This finding was confirmed by ellipsometric measurements, demonstrating the isotropy of the \\ $\mathrm{PhC}$ resonances. Such negative index $\mathrm{PhC}$ materials may be of use in biosensing applications.
}

Light: Science \& Applications (2012) 1, e42; doi:10.1038/lsa.2012.42; published online 21 December 2012

Keywords: diffraction gratings; metamaterials; photonic crystals

\section{INTRODUCTION}

Photonic crystals (PhCs) have reshaped the landscape of photonic devices $^{1}$ with such early applications as waveguides ${ }^{2,3}$ and resonant cavities, ${ }^{4}$ as well as multiplexing. ${ }^{5}$ The power of $\mathrm{PhCs}$ and their unusual properties arises from the optical bandgap, which can be tailored by design. Recently, PhCs have been used more widely as a metamaterial with an effective negative refractive index. ${ }^{6-10}$ Light propagating in such a metamaterial can undergo a drastic change in group velocity, causing the light to bend away from the usual direction that is observed with a conventional refracting medium. Owing to the fine control over the optical bandgap of the $\mathrm{PhC}$, the effective refractive index can exhibit optical antimatter behavior. ${ }^{7}$ For example, a set of circular holes etched vertically into a silicon slab in a hexagonal arrangement can produce an effective resonant refractive index of $n_{\text {eff }}=-1$ for light propagating along the length of the slab (perpendicular to the direction of the holes). Such a metamaterial strongly couples incoming light and can be used to transmit data with minimum losses over millimeter distances for lab-on-a-chip applications. ${ }^{8}$

The reflection spectra of patterned surfaces have been widely studied since the discovery of Wood anomalies in metallic gratings. ${ }^{11}$ In particular, Fano found a clear connection between the narrow anomalies and surface wave excitation that distinguished broad and sharp anomalies. ${ }^{12}$ The theoretical understanding of this phenomenon has been provided by Hessel and Oliner. ${ }^{13}$ Similarly, the resonances in $\mathrm{PhC}$ slabs have been analyzed in reflection and transmission. ${ }^{14}$ These resonances have been used to reconstruct the band structure ${ }^{15}$ and equifrequency surface ${ }^{16}$ of $\mathrm{PhCs}$. Similar to surface plasmon polariton (SPP) resonances, the resonant anomalies allow for the propagation of otherwise forbidden modes. ${ }^{15}$ Other analogies with SPP behavior arise for $\mathrm{PhCs}$ at frequencies where the refractive index turns negative. In this paper, we present an experimental study of the resonance effects directly connected with negative refractive index properties of $\mathrm{PhC}$. Using standard ellipsometric measurements of the amplitude $\psi$ and phase $\Delta$ of the ratio between the linear polarization reflection coefficients, we demonstrate that the change in the dielectric function of negative index $\mathrm{PhC}$ is similar to the change in the dielectric constant of a plasmonic structure. ${ }^{17}$ Similar to the Fano resonances, ${ }^{17,18}$ the resonances in the $\psi$ spectrum corresponding to a $180^{\circ}$ phase change are slightly asymmetric. A Lorentz model description of the dielectric function shows that only a superficial layer of the $\mathrm{PhC}$ slab is involved in the resonance effect in the wavelength range of the negative refractive behavior, demonstrating that such resonances are insensitive to the orientation of the slab. Therefore, such guided modes correspond to an isotropic negative refractive index in PhCs.

\section{MATERIALS AND METHODS}

In this study, we experimentally analyze the three-dimensional problem of a light beam on a PhC slab with an incident wave vector $\vec{k}$ out of the slab plane. The $2 \times 2 \mathrm{~mm} \mathrm{PhC}$ is realized with high-resolution lithography on a silicon-on-insulator wafer that has a $1.5 \mu \mathrm{m}$ top silicon layer and is perforated by a set of circular holes of radius $r=180 \mathrm{~nm}$ arranged in a hexagonal lattice with parameter $a=472 \mathrm{~nm}$ (Figure 1a). Vertical light confinement is provided by refractive index contrast at the silicon/oxide and silicon/air interfaces. Under these conditions, the top layer of silicon is sufficient to ensure an effective two-dimensional behavior. For a resonant wavelength of $\lambda=1.55 \mu \mathrm{m}$, the effective refractive index of the fundamental mode supported by a silicon uniform slab waveguide of this thickness is $n_{\text {eff }}($ TE,TM) $=3.45$, which is equal to the bulk silicon value for both polarizations. The condition for the negative refractive index,

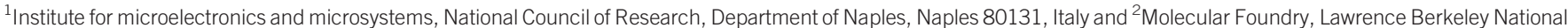
Laboratory, Berkeley, CA, USA

Correspondence: Dr V Mocella, Institute for microelectronics and microsystems, National Council of Research, Department of Naples, Naples 80131, Italy

E-mail: vito.mocella@na.imm.cnr.it

Received 21 March 2012; revised 30 July 2012; accepted 13 August 2012 
a
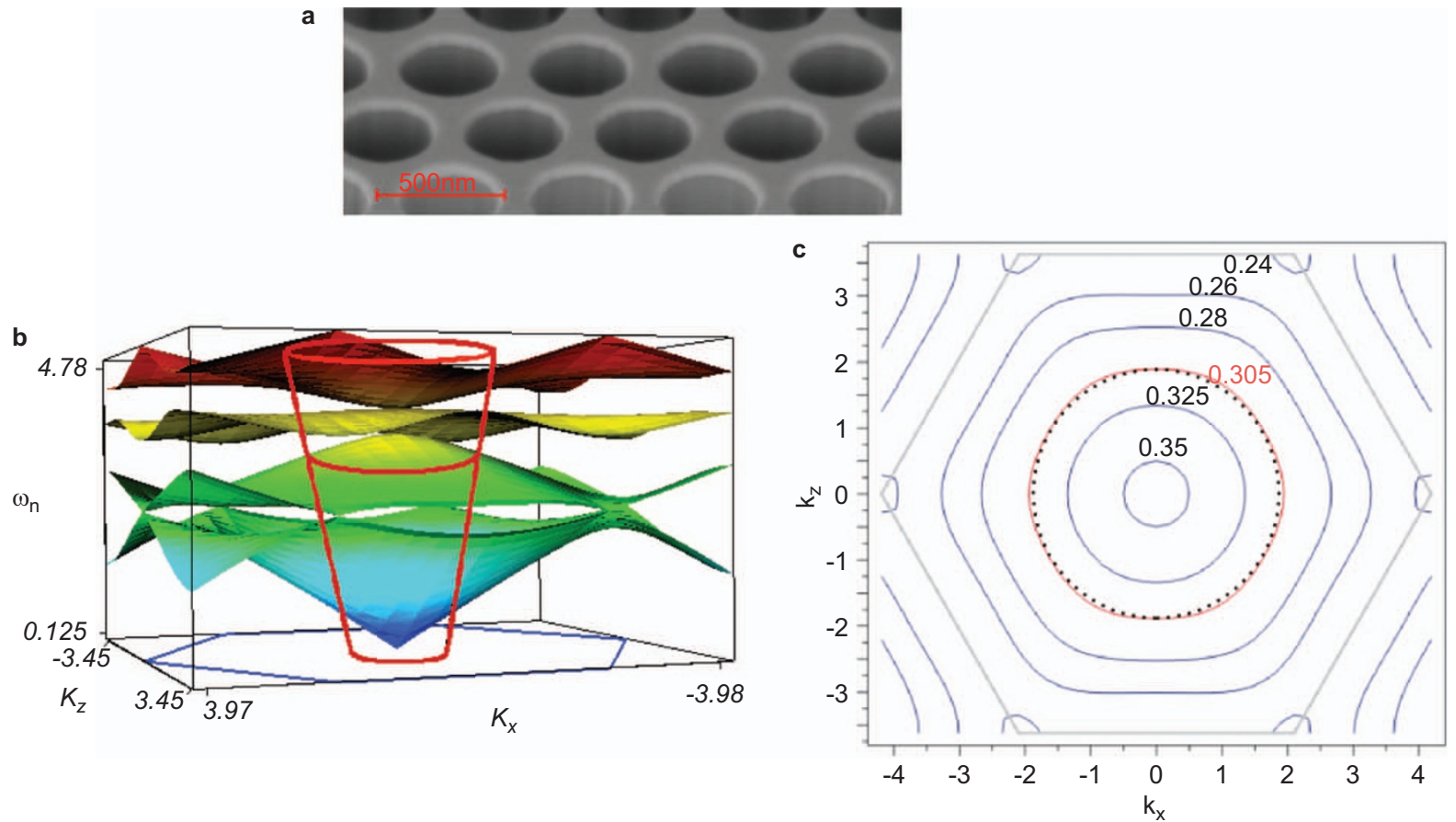

Figure 1 The scanning electron microscope image of the PhC is shown in (a) with the corresponding dispersion relation given in (b) and (c). The red line corresponds to the light line in air (or vacuum). The coupling between the incident wave in air and inside the PhC occurs when the dispersion relation intersects the light line, as shown in (b) for a normalized frequency $\omega_{\mathrm{n}}=0.305$ at a resonant wavelength $\lambda=1.55 \mu \mathrm{m}$ for the TM-polarized incident light. PhC, photonic crystal; TM, transverse magnetic.

$\vec{v}_{g} \cdot \vec{k}<0$, is satisfied around the normalized frequency $\omega_{\mathrm{n}}=a /$ $\lambda=0.305$ when the group velocity $\vec{v}_{\mathrm{g}} \equiv \partial \omega / \partial \vec{k}$ is directed inwards, pointing to the origin of the first Brillion zone. For light that is polarized perpendicular to the surface of the $\mathrm{PhC}$ slab (i.e., transverse magnetic with the electric field along the direction of the circular holes), the equifrequency surface exhibits a circular shape (Figure $1 \mathrm{~b}$ and $1 \mathrm{c}$ ) corresponding to an isotropic medium with an effective index of refraction $n_{\mathrm{eff}}=-1$.

For a plane wave incident on the PhC slab, momentum conservation requires that both the parallel and perpendicular components of the incident wave vector be conserved for efficient coupling to the guided modes in the $\mathrm{PhC}$ slab. However, only the parallel component is conserved across the air/PhC interface, so the perpendicular component is determined according to the dispersion relation shown in Figure 1c. Perpendicular component is conserved only in special case such as the normalized frequency $\omega_{\mathrm{n}}=0.305$ does the light couple efficiently to the guided mode inside the PhC. In this case, a resonance arises from the interference between the wave refracted inside the $\mathrm{PhC}$ slab Fabry-Pérot structure and the guided mode excited inside the $\mathrm{PhC}$ by the incident wave. This interference gives rise to guided resonances that have the typical asymmetric shape of Fano resonances, associated with combinations of wide and narrow resonances. ${ }^{14}$

\section{RESULTS AND DISCUSSION}

Similar to the work by Kravets et al., ${ }^{17}$ periodic structure resonances can be analyzed by means of ellipsometric measurements recognized as a peak in the $\psi$ spectrum corresponding to a $180^{\circ}$ jump in the $\Delta$ phase spectrum, where $\psi$ and $\Delta$ are defined by

$$
\frac{r_{\mathrm{p}}}{r_{\mathrm{s}}}=\tan \psi e^{-\mathrm{i} \Delta}
$$

where $r_{\mathrm{p}}$ and $r_{\mathrm{s}}$ are the complex Fresnel coefficients. In Figure 2, a sharp resonance is clearly visible as a peak in the $\psi$ spectrum and a corresponding $180^{\circ}$ shift in the phase for an incident wavelength $\lambda=1.55 \mu \mathrm{m}$.

To maximize the signal-to-noise ratio, the angle of incidence was set to $70^{\circ}$, and the sample was rotated in-plane by an angle $\theta$ between the incident plane and the $\Gamma-K$ symmetry direction in the plane of the $\mathrm{PhC}$, as indicated by the red arrow in the inset of Figure 2. Owing to the sixfold symmetry of the hexagonal lattice, measurement of the coupling for angles $\theta$ in the range of $\pm 15^{\circ}$ covers the entire irreducible Brillouin zone. For all angles of rotation $\theta$, a peak is observed in the $\psi$ spectrum with a corresponding $180^{\circ}$ shift in the $\Delta$ spectrum at a resonant wavelength $\lambda=1.55 \mu \mathrm{m}$. Figure 2 shows the spectra for three representative angles $\theta$.

The spectrum shown in Figure 2 can be split into two regions: the peaks in the $1.4 \mu \mathrm{m}$ band can be used to measure the $\mathrm{PhC}$ band structure; ${ }^{15,16}$ however, above $1.5 \mu \mathrm{m}$, the spectrum is independent of $\theta$ and isotropic, suggesting that the $\mathrm{PhC}$ resonances might be useful for sensing applications. This isotropic property of the resonance is further proof of the connection between the resonance and isotropic negative refractive index behavior in Figure 1c.

Characterizing the medium response by a generalized dielectric function $\varepsilon(\omega, k)$, i.e., using a spatial dispersion approach, the isotropic behavior of this portion of the spectrum is equivalent to a simple $k^{2}$ dependence: $\varepsilon(\omega, k)=\varepsilon(\omega)+\alpha k^{2}$. This characteristic has been shown to be equivalent to a description in terms of $\varepsilon_{\text {eff }}$ and $\mu_{\text {eff }}$, where $1 / \mu_{\mathrm{eff}}(\omega)=1-\omega^{2} \alpha(\omega){ }^{19}$

Ellipsometric analysis of the silicon-on-insulator sample yields the optical constants and thickness of the top PhC layer of the sample. ${ }^{20}$ We consider a non-local homogenization, as successfully applied by Vynck et al., ${ }^{21}$ on a model proposed by Silveirinha. ${ }^{22}$ The sketch of the multilayer sample and the results of the fit analysis are shown in Figure 3. In this analysis, the sample was treated as a multilayer system by imposing the order and the material of the layers. A fit of the $\psi$ and $\Delta$ spectra yields information for both the structure and single $\mathrm{PhC}$ 

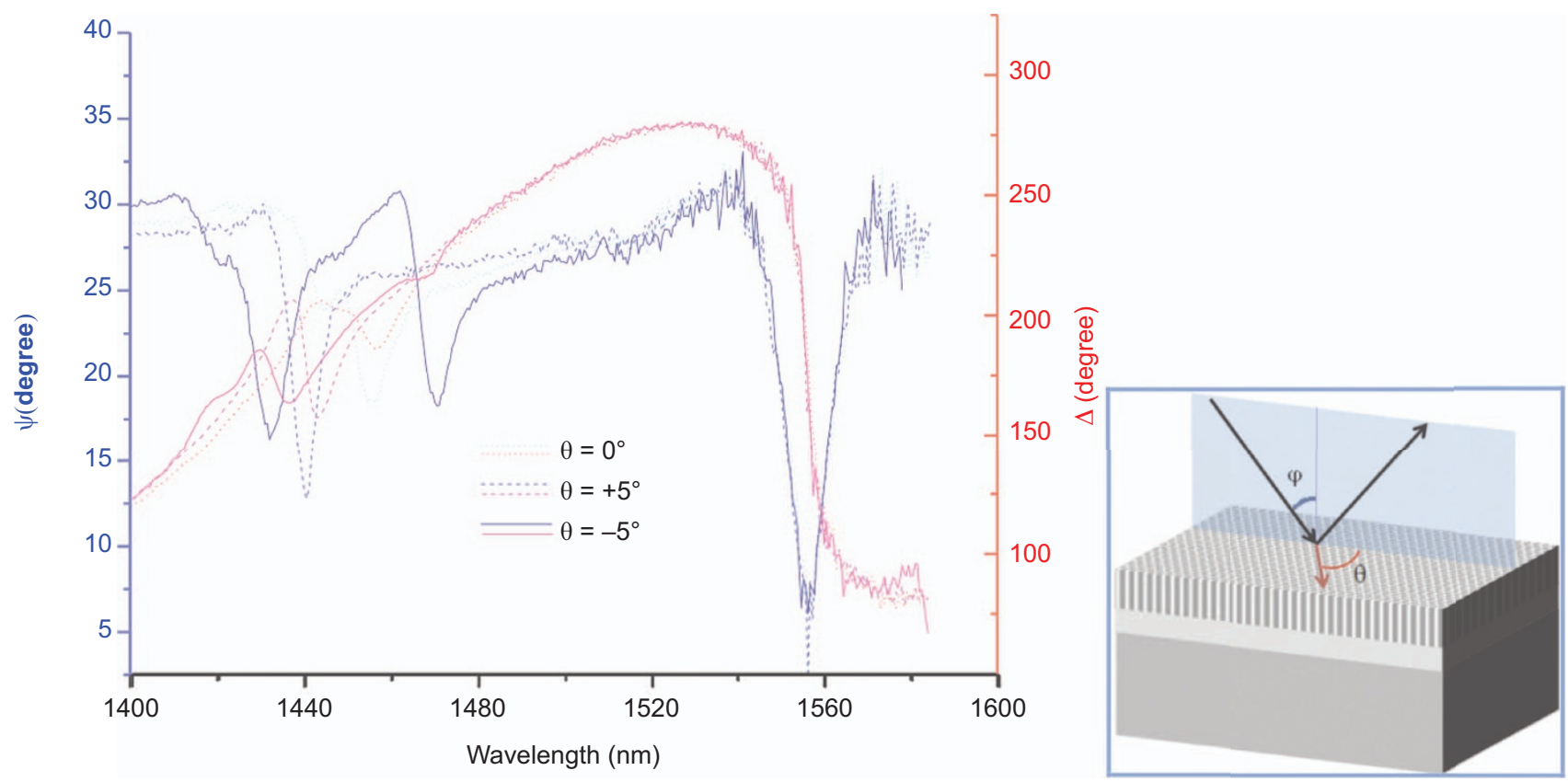

Figure 2 Ellipsometric measurements: blue tone lines define $\psi$ spectra, and red tone lines define $\Delta$ spectra. The peak in $\psi$ and the $180^{\circ}$ jump in the $\Delta$ spectrum appear at $\lambda_{0}=1.55 \mu \mathrm{m}$ for all $\theta$ angles. Inset: measurement geometry: $\varphi$ is the angle between the incident wave vector and the normal to the surface in the incident plane; $\theta$ is the angle between the incident plane and the $\Gamma-K$ symmetry direction (red arrow) in the PhC plane. PhC, photonic crystal.

layer at approximately $1.55 \mu \mathrm{m}$. The $\mathrm{PhC}$ structure was modeled by three layers: (i) the top $30 \mathrm{~nm}$ was modeled as a generic Lorentz oscillator, equation (2), for transverse magnetic polarization or as an Si-air mix (46.23\% Si and 53.77\% air) for transverse electric polarization; (ii) $1.47 \mu \mathrm{m}$ of the Si-air mixture; and (iii) $160 \mathrm{~nm}$ of pure Si corresponding to the under-etch of the PhC. The PhC structure was then modeled to reside on the $870 \mathrm{~nm}$ silicon dioxide layer on the crystalline Si wafer. The layered structure model is sketched in Figure $3 b$, and the parameters are summarized in Figure $3 c$, with the fit parameters indicated by the symbol ' $\mathrm{F}$ '.

$$
\varepsilon(\omega)=\frac{f \omega_{0}^{2}}{\omega_{0}^{2}-\omega^{2}+i \gamma \omega}+\varepsilon_{\infty}
$$

In equation (2), $\varepsilon_{\infty}$ is the high-frequency dielectric constant, $\omega_{0}$ is the resonant frequency and $\gamma$ is the damping constant.

The fit analysis provides a Lorentz oscillator confined in a top surface layer that is no more than $30 \mathrm{~nm}$ thick. The Lorentz parameters are: $f=0.40, \omega_{0}=0.804 \mathrm{eV}, \gamma=-0.018 \mathrm{eV}$ and $\varepsilon_{\infty}=4.24$. With these parameters, the real portion of the dielectric constant given by equation (2) shows negative values for wavelengths in the range of negative $\mathrm{PhC}$ behavior (Figure 4). In particular, at $\lambda=1548.7 \mathrm{~nm}, \varepsilon=-1$, which is in agreement with the $\mathrm{PhC}$ design. These results confirm that only a thin top-surface layer at the air/PhC interface is involved in the resonant negative refraction behavior of the $\mathrm{PhC}$. These results suggest that a mode is confined to a thin interface region in strong analogy to SPP modes. A further analogy with SPP behavior is observed in the asymmetric shape of the experimental $\psi$ spectrum, typical of Fano resonance. ${ }^{23}$

The resonant coupling of light at an incident of $1.55 \mu \mathrm{m}$ on the $\mathrm{PhC}$ metamaterial at an oblique angle of incidence is due to the momentum match in the dispersion relation, as shown in Figure 1. The resonance is confined to a thin top surface layer. We expect the resonance wavelength to be strongly dependent on the surrounding environment, and this property suggests that such $\mathrm{PhC}$ metamaterial can be used for sensing applications that are analogous to the plasmonic biosensing, ${ }^{24}$ with a benefit of having significantly lower signal losses due to the lack of any metallic components.

\section{CONCLUSIONS}

A PhC metamaterial was found to exhibit narrow bandwidth resonant guided modes in a top surface layer with a thickness of approximately $30 \mathrm{~nm}$. These resonances resemble their plasmonic counterparts. In the frequency range in which the effective refractive index of the $\mathrm{PhC}$ is negative, the resonances corresponding to these propagating modes were clearly identified by conventional ellipsometric analysis. This resonant phenomenon is the dielectric equivalent to Wood anomalies and is isotropic with respect to $\mathrm{PhC}$ orientation, which is in agreement with the propagation of guided modes in an isotropic negative refractive index metamaterial.

\section{AUTHOR CONTRIBUTIONS}

$\mathrm{PD}$ acquired data, had a role in interpreting the results and drafted the manuscript. MG acquired data, contributed to interpreting the results and revised the manuscript. IR contributed to interpreting the results and revised the manuscript. SC fabricated the samples, contributed to interpreting the results and revised the manuscript. VM conceived and designed the work that led to the submission, had an important role in interpreting the results and drafted the manuscript.

\section{ACKNOWLEDGMENTS}

Portions of this work were completed as part of a user project at the Molecular Foundry, Lawrence Berkeley National Laboratory, which is supported by the US Department of Energy under contract DE-AC02-05CH11231. We gratefully acknowledge Aleksandr Polyakov for his suggestions in critically revising the manuscript. This work was partially supported by Italian National Operative Programs PON01_02782, PON01_01525 and PON01_00375. 

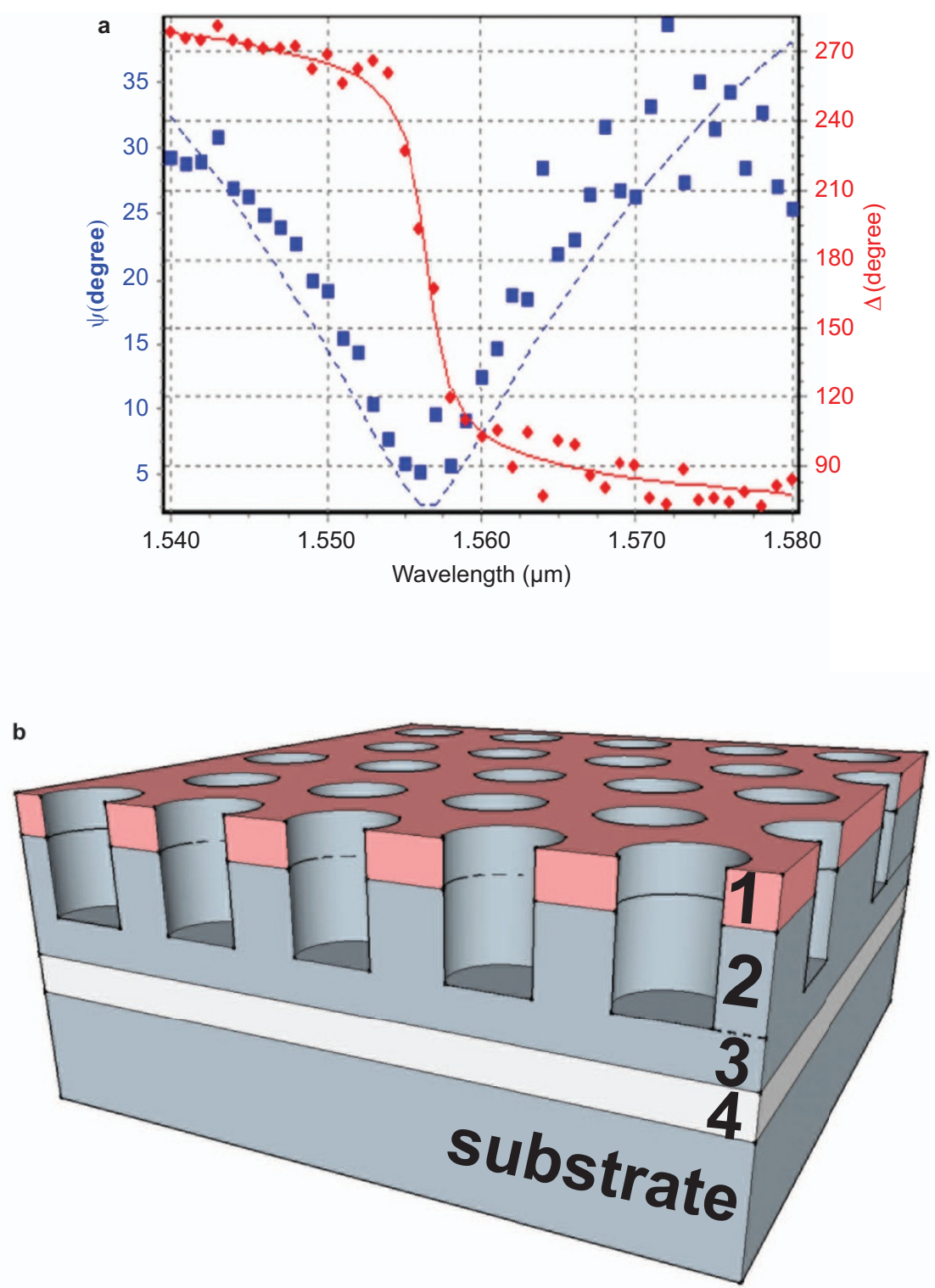

\begin{tabular}{|c|c|c|c|c|}
\hline Layer & Thickness (nm) & Polarization & \multicolumn{2}{|c|}{ Material } \\
\hline \multirow{2}{*}{1} & \multirow{2}{*}{$30(F)$} & TE & \multicolumn{2}{|c|}{ Lorentz Oscillator (F) } \\
\hline & & TM & C-Si $46.23 \%$ & Air $53.77 \%$ \\
\hline 2 & $1470(F)$ & both & C-Si $46.23 \%$ & Air $53.77 \%$ \\
\hline 3 & $160(F)$ & both & \multicolumn{2}{|c|}{ C-Si } \\
\hline 4 & $870(F)$ & both & \multicolumn{2}{|c|}{$\mathrm{SiO}_{2}$} \\
\hline & ubstrate & both & \multicolumn{2}{|c|}{$\mathrm{C}-\mathrm{Si}$} \\
\hline
\end{tabular}

Figure 3 Results of the analysis. (a) Experimental data for $\psi$ (blue square) and $\Delta$ (red circle) spectra with the resulting fit (bold lines). (b) Sketch of the multilayer sample model. (c) Results of the fit: the first column on the left provides the number order of the layer, the second column provides the resulting thickness and the last column provides the materials with the composition percentage. The symbol ' $F$ ' indicates an analyzed parameter in the fit. 


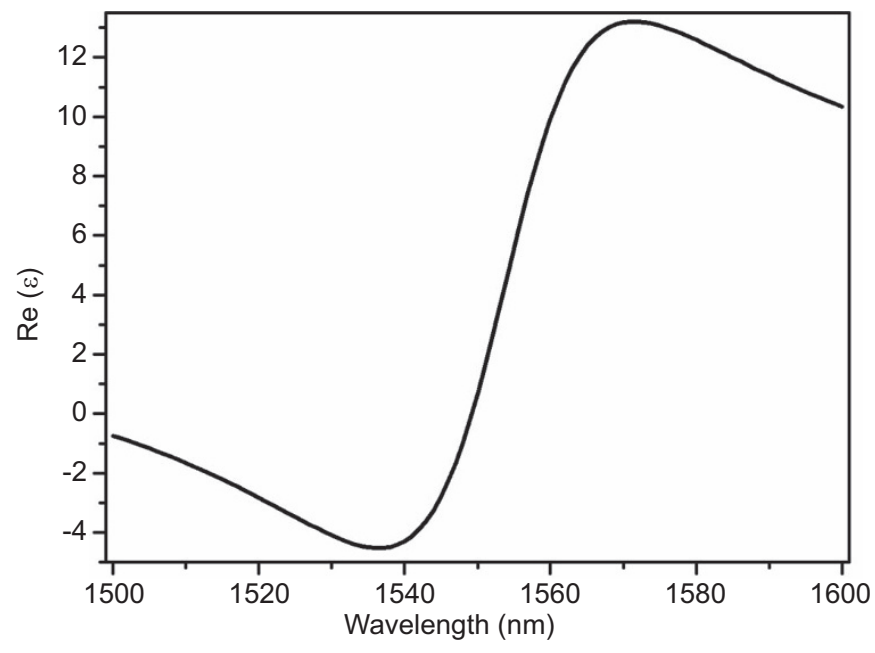

Figure 4 Real portion of the dielectric function $\varepsilon(\omega, k)$ of the Lorentz oscillator. For $\lambda=1.5487 \mu \mathrm{m}, \varepsilon=-1$, which is in agreement with the theoretical model.

1 Joannopulos JD, Mead RD, Winn JN. Photonic crystal: molding the flow of light Princeton, NJ: Princeton University Press, 1995.

2 Jamois C, Wehrspohn RB, Andreani LC, Herman C, Hess 0 et al. Silicon-based twodimensional photonic crystal waveguides. Phot Nanostr 2003; 1: 1-13.

3 Sakoda K. Optical properties of photonic crystal. Berlin: Springer-Verlag, 2001.

4 Johnson SG, Joannopoulos JD. Photonic crystals - the road from theory to pratice. Boston, MA: Kluwer Academic Publishers, 2003.

5 Dardano P, Moretti L, Mocella V, Sirleto L, Rendina I. Investigation of a tunable Tshaped waveguides based on a silicon 2D photonic crystal. J Opt A: Pure App/ Opt 2006; 8: S554.

6 Pendry JB, Smith DR. Reversing light with negative refraction. Phys Today 2004; 57: 37-43.

7 Mocella V, Cabrini S, Chang A, Dardano P, Moretti L et al. Self-collimation of light over millimeter-scale distance in a quasi-zero-average-index metamaterial. Phys Rev Let 2009; 102: 133902.
8 Smith $\mathrm{H}$, Hawkin AR. The photonic integration of non-solid media using optofluidics. Nat Photon 2011; 5: 598-604.

9 Notomi M. Theory of light propagation in strongly modulated photonic crystals: refractionlike behavior in the vicinity of the photonic band gap. Phys Rev $B$ 2000; 62: 10696-10705.

10 Mocella V, Dardano P, Rendina I, Cabrini S. An extraordinary directive radiation based on optical antimatter at near infrared. Opt Express 2010; 18: 25068-25074.

11 Wood RW. On the remarkable case of uneven distribution of light in a diffraction grating spectrum. Philos Mag 1902; 4: 396-402.

12 Fano U. The theory of anomalous diffraction gratings and of quasi-stationary waves on metallic surfaces (Sommerfeld's waves). J Opt Soc Am 1941; 31: 213-222.

13 Hessel A, Oliner AA. A new theory of Wood's anomalies on optical gratings. Appl Opt 1966; 4: 1275-1299.

14 Fan S, Joannopoulos JD. Analysis of guided resonances in photonic crystals. Phys Rev B 2002; 65: 235112.

15 Astratov VN, Whittaker DM, Culshaw IS, Stevenson RM, Skolnick MS et al. Photonic band-structure effects in the reflectivity of periodically patterned waveguides. Phys $\operatorname{Rev} B$ 1999; 60: R16255.

16 Peyrade D, Torres J, Coquillat D, Legros R, Lascaray JP et al. Equifrequency surfaces in GaN/sapphire photonic crystals. Physica E 2003; 17: 423-425.

17 Kravets VG, Schedin F, Grigorenko AN. Extremely narrow plasmon resonances based on diffraction coupling of localized plasmons in arrays of metallic nanoparticles. Phys Rev Lett 2008; 101: 087403

18 Luk'yanchunk B, Zheludev NI, Maier SA, Halas NJ, Ordlander P et al. The Fano resonance in plasmonic nanostructures and metamaterials. Nat Mater 2010; 9: 707.

19 Agranovich VM, Shen YR, Baughman RH, Zakhidov AA. Linear and nonlinear wave propagation in negative refraction metamaterials. Phys Rev B 2004; 69: 165112.

20 Fujiwara H. Spectroscopic ellipsometry: principles and applications. Chichester: John Wiley \& Sons, 2007

21 Vynck K, Felbacq D, Centeno E, Căbuz AI, Cassagne D et al. All-dielectric rod-type metamaterials at optical frequencies. Phys Rev Lett 2009; 102: 133901.

22 Silveirinha MG. Nonlocal homogenization model for a periodic array of $\varepsilon$-negative rods. Phys Rev E 2006; 73: 046612

23 Rybin MV, Khanikaev AB, Inoue M, Samusev KB, Steel MJ et al. Fano resonance between Mie and Bragg scattering in photonic crystals. Phys Rev Lett 2009; 103 : 023901.

24 McFarland AD, van Duyne RP. Single silver nanoparticles as real-time optical sensors with zeptomole sensitivity. Nano Lett 2003; 3: 1057-1062.

(c) This work is licensed under a Creative Commons

SOMERIEHISHESERVED Attribution-NonCommercial-NoDerivative Works 3.0

Unported License. To view a copy of this license, visit http:// creativecommons.org/licenses/by-nc-nd/3.0 